tool to the object), unrecognisable errors, and no response errors.

When using her left hand she was correct on nine of $10(90 \%)$ intransitive gestures making one unrelated content error and she was correct on three of 20 (15\%) transitive gestures making 19 spatial, three temporal, and two related content errors. When using her right hand she was correct on eight of $10(80 \%)$ intransitive gestures making two spatial and one temporal error and she was correct on four of $20(20 \%)$ transitive gestures making 22 spatial and four temporal errors.

The gesture to command task was also given to 12 normal men and women in thei late $60 \mathrm{~s}$. They performed 10 of $10(100 \%)$ intransitive gestures and 17 to 20 of 20 transitive gestures correctly ( $85 \%$ to $100 \%$, mean $18.9,94.5 \%)$. Normal subjects made 11 temporal errors and two spatial errors.

When given the actual tool to use, she was correct on eight of $20(40 \%)$ trials when using her left hand and nine of 20 $(45 \%)$ trials when using her right hand.

She was given the name of the tools and intransitive gestures from the gesture to command subtest. For each item she was presented with two gestures performed by the examiner, one the correct gesture and one an incorrect gesture (spatial error), and she was asked to choose the gesture that best fitted the item. Each set of gestures was performed twice. She was correct on 25 of $30(82 \cdot 5 \%)$ trials. Five age, education, and sex matched normal controls were correct on 28 to 30 of $30(93 \%$ to $100 \%$, mean $29 \cdot 2(97 \%))$ trials.

The patient was shown 50 two and three step non-meaningful gestures involving the hand and arm on videotape. Each gesture was shown twice and she was asked to imitate the gesture. She was unable to imitate any of the 50 gestures correctly.

She was given a rotary pursuit task to assess her motor skill learning and did not show any motor skill learning with either hand.

She showed an ideomotor limb apraxia as the result of a left posterior cerebral artery infarct. The praxis system is a network that anatomically includes the parietal lobes, the frontal lobes, the white matter connections between them (both intrahemispheric and interhemispheric (corpus callosum)), and possibly the basal ganglia. ${ }^{2}$ The neural structures subserving the praxis network are fed by the branches of the middle and anterior cerebral arteries whereas apraxia is not typically reported after posterior cerebral artery infarcts. ${ }^{3}$

Her apraxia was characterised by impaired production of meaningful and non-meaningful gestures with relative preservation of gesture recognition. Her apraxia improved but was still present when using tools. Her apraxia was more severe for transitive than for intransitive gestures. She also had absent motor learning on a rotary pursuit task as has been found in other apraxic patients. ${ }^{4}$

Damage to the occipital or temporal lobes has not been implicated in limb ideomotor apraxia. Apraxia secondary to dominant thalamic injury has been reported although not often investigated. Most of these cases have been thalamic hemorrhages that may have produced apraxia secondary to compression of non-thalamic structures. Graff-Radford et $a \bar{l}$ described three patients with thalamic infarcts in the territory of the tuberothalamic artery who showed limb apraxia.

The pulvinar has connections with the inferior parietal cortex and lateral prefronta cortex, cortical structures important for praxis, thus making it a likely candidate for contributing to gesture processing. Based on its connections, we suggest that the pulvinar may participate in the selection of the movement representation, the translation of the representation into spatiotemporal coordinates, and the mediation of feedback for gesture monitoring.

The thalamus has been posited to function as a gated relay to filter out irrelevan information and may selectively engage relevant cortical neurons thereby creating transient cortical networks (Nadeau and Crosson, personal communication). Thalamic function is dependent on its thalamocortical connections for input and output and is regulated by the frontal lobe-inferior thalamic peduncle-nucleus reticularis-centromedian system. Therefore apraxia could arise from damage to the pulvinar as the result of its connections with the parietal and frontal cortex as shown by our patient or from damage to the frontal lobe-inferior thalamic peduncle-nucleus reticularis-centromedian system as shown by the patients with tuberothalamic infarcts reported by Graff-Radford et al. ${ }^{5}$

Further cases of thalamic lesions will help define the role of the thalamus in the praxis network.

$$
\begin{array}{r}
\text { JEFFREY E SHUREN } \\
\text { Department of Neurology, } \\
\text { University of Cincinnati Medical Center, } \\
\text { Cincinnati, Ohio, USA } \\
\text { LYNN M MAHER } \\
\text { Gainesville, Florida, USA } \\
\text { Kepartment of Veterans' Affairs Medical Center, } \\
\text { KENNETH M HEILMAN } \\
\text { Center for Neuropsychological Studies, } \\
\text { Department of Neurology, University of Florida } \\
\text { College of Medicine, Florida, USA }
\end{array}
$$

Correspondence to: Dr Jeffrey Shuren, University of Cincinnati Medical Center, Department of Neurology, 4010 Medical Sciences Building (ML 525), 231 Bethesda Avenue, Cincinnati, OH 45267-0525, USA.

1 Rothi LJG, Mack L, Verfaellie M, Brown P, Heilman KM. Ideomotor apraxia. error patHeilman KM. Ideomotor apraxia: error
tern analysis. Aphasiology 1988;2:381-8.

2 Kertesz A. Apraxia and aphasia. Anatomical and clinical relationship. In Roy EA, ed. Neuropsychological studies of apraxia and related disorders. New York: North Holland, 1985:163-78.

M, ed. Principles of behavioral neurology. Philadelphia: FA Davis, 1988.

4 Heilman KM, Schwartz HD, Geschwind N. Defective motor learning in ideomotor apraxia. Neurology 1975;25:1018-20.

5 Graff-Radford NR, Eslinger PJ, Damasio AR, Yamada T. Nonhemorrhagic infarction of Te thalam: becior anatomic, and physiologic correlates. Neurology 1984;34:14-23.

\section{Anomia for proper names after left thalamic infarct}

Proper names constitute the category of words most exposed to the "tip of the tongue" phenomenon. ${ }^{1}$ Older people are more susceptible to this difficulty, which is their most common spontaneous complain regarding memory performance. ${ }^{2}$ A pronounced impairment in naming faces is often noted in Alzheimer's disease, ${ }^{3}$ but has only been reported rarely as an isolated deficit after focal cerebral lesions. ${ }^{4-9}$ We describe a patient who presented an impairment in retrieving proper names as the only linguistic deficit after left thalamic stroke.

The patient was a 59 year old ambidextrous man, working as a senior executive. He had a history of migraine, hypertension, and hypercholesterolaemia. One morning his secretary noticed that he was abnormally drowsy and slow and had moderate speech difficulties. These abnormalities disappeared within 24 hours. When the patient consulted a few days later, he only had vague recollections of this initial day. He spontaneously complained, however, of severe difficulties in retrieving the names of familiar people. When asked to enumerate recent French prime ministers from memory, he could provide accurate descriptions of their physical aspect, city of origin, and political tendency, but could not find their names. He was unable to find the names of two close members of his own family. There was no motor, sensory, oculomotor, or visual defect, and no aphasia, apraxia, or spatial neglect.

Cerebral MRI (figure) showed a left thalamic infarct in the territory of the anterior polar artery, affecting the ventral anterior and ventral lateral nuclei, the mamillothalamic tract, and possibly part of the dorsomedial nucleus. PET with ${ }^{15} \mathrm{O}$ labelled $\mathrm{H}_{2} \mathrm{O}$ showed a diffuse and moderate hypometabolism of the left hemisphere.

The patient scored at ceiling level in all subtests of the Boston diagnostic aphasia examination. On the revised Wechsler memory scale, he had a visual index of 106 , but a verbal index of $79(1.2 \mathrm{SD}$ below the mean).

To assess the patient's ability to name people, he was presented with a set of 45 pictures of famous faces and asked to name them. He could name only six of these, performing significantly worse than five control subjects matched for age and educational level $(t(4)=3.36, p<0.03)$. He identified most targets, however, as evidenced by accurate biographical comments. Moreover, in $78 \%$ of the trials on which he declared having identified the target face without being able to retrieve the name, the patient could produce the correct name as soon as he was provided with its initial syllable. Two observations further support the hypothesis of an impaired name retrieval with intact face perception and recognition. Firstly, the patient scored in the normal range in Benton's facial recognition test. Secondly, as noted before, his word finding deficit was also apparent in non-visual tasks such as spontaneous speech and naming on definition.

Although the patient had major difficulties retrieving proper names for output, comprehension was apparently normal. On verbal presentation of their name, he provided a precise verbal characterisation of 14 of 15 famous people, of which he could name only three when confronted with a picture of their face.

To evaluate the selectivity of the naming deficit, the patient was asked to name 80 line drawings of objects belonging to 10 semantic categories, as well as 12 patches of colour, and 20 body parts pointed out on the experimenter's body. His performance was flawless except for two items, which he could readily name as soon as he was provided with the first syllable. To establish whether all kinds of proper names were 


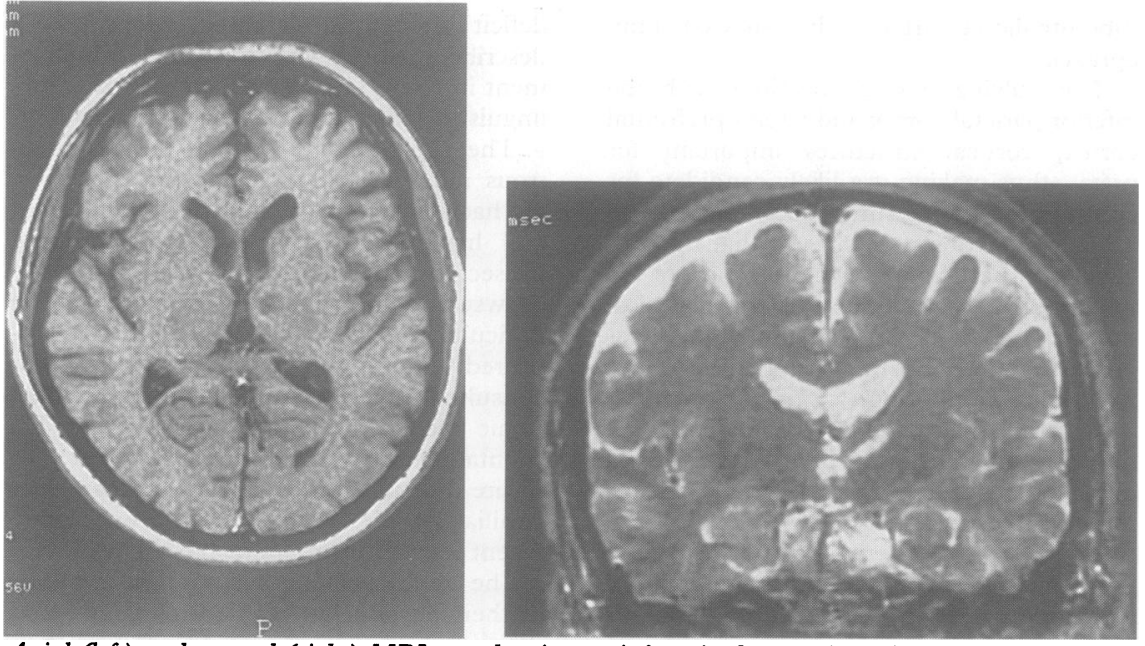

Axial (left) and coronal (right) MRI cuts showing an infarct in the anterior polar territory of the left thalamus, affecting the ventral anterior and ventral lateral nuclei, the mammillothalamic tract, and possibly part of the dorsomedial nucleus.

equally affected, the patient was presented with a map of Europe and asked to name all the countries and their capital cities. He easily named 24 of 24 countries and 23 of 24 capitals. When presented with a map of France, he could name 15 of 16 cities whose locations were marked by a dot.

In summary, a patient presented a selective deficit in retrieving names of familiar people, after left thalamic stroke. His additional deficit of verbal memory should probably be ascribed to the lesion of the mammillothalamic tract and dorsomedial nucleus, the role of which in anterograde memory has been clearly established. The naming deficit, which constitutes the core of this observation, raises three related questions: (1) What was the functional mechanism underlying the deficit? (2) How does the impairment relate to the role of the thalamus in language processes? (3) Why does the brain process people's names differently from other words?

Producing familiar people's names when confronted with a picture of their faces may be impaired at any one of three successive stages, each correlated with a more or less precisely identified lesion site. Firstly, prosopagnosic patients with a right occipitotemporal lesion are unable to access stored canonical representations of faces, and hence cannot retrieve any attached knowledge, including proper names. Secondly, patients with focal or degenerative temporal lesions may show reduced face naming abilities as a result of a generalised loss of semantic information concerning specific subjects. The present case does not correspond to either of these two patterns, as the patient could adequately retrieve information relevant to subjects he was unable to name. Thirdly, several patients have been described who showed normal recognition of familiar faces and normal access to the associated knowledge, but were unable to retrieve the corresponding name in the verbal output system. ${ }^{4-9}$ This is clearly the case in the patient we have described, whose deficit can be construed as a category specific word finding problem, or anomia.

Cases of anomia for proper names, however, are not identical in all respects. Whereas some patients showed a nearly total deficit, extending beyond people's names to categories such as cities, countries, and rivers, ${ }^{56}$ others had a less severe impairment, generally restricted to people's names. ${ }^{6910}$ Furthermore, the precise mechanism underlying anomia was probably not the same in all cases. In our patient, as well as in the patient studied by Lucchelli and De Renzi, ${ }^{7}$ anomia could be dramatically reduced when the examiner provided a phonological cue. This indicates that knowledge of word forms was not lost but, rather, was difficult to activate to a sufficient level. In other cases, the absence of any effective phonological cueing is compatible with a destruction of the lexicon for proper names. ${ }^{56} \mathrm{~A}$ third possible mechanism was recently put forward by Shallice and Kartsounis, ${ }^{9}$ whose patient had a long standing temporal glioma. The authors argued that the impairment actually corresponded to an anterograde memory defect restraining the ability to create new associations between meaning and words.

Although the precise contribution of the thalamus to naming remains poorly understood, it is thought to play an important part in activating and coordinating the left temporal lobe, where there is some evidence that proper names may be stored, ${ }^{10}$ and the left frontal language area, which is crucial to the production of any verbal output. ${ }^{11}$ This hypothesis is compatible with the projections from the ventral anterior nucleus to the frontal lobe, and from the dorsomedia nucleus to the anterior and posterior language areas. In the present case, as well as in the other reported patient with thalamic anomia for proper names, ${ }^{7}$ performance was substantially improved by phonological cueing, whereas this was not the case in similar patients with temporal lesions. ${ }^{56}$ It may thus be speculated that phonological cueing compensated for the lack of a sufficient activation of the output processes that resulted from a thalamic lesion.

Whereas it seems possible to identify the mechanisms underlying anomia, it is no obvious in the first place why the brain should process proper names differently from other kinds of words. Two main types of hypothesis have been proposed. Firstly, this selectivity could reflect the internal organisation of the semantic system. On this account, anomia would result from an impaired access to the output lexicon from the semantic store, restricted to the subset of semantic memory concerned with familiar people. This deficit would be equivalent to selective anomia for other semantic categories, such as has been reported for body parts, fruits and vegetables, etc. ${ }^{12} \mathrm{~A}$ second hypothesis puts an emphasis on the fact that proper names may be considered as verbal labels arbitrarily linked to a given subject, which they identify without providing any kind of semantic information. By contrast, common names entail some description of the item to which they are attributed. For instance, the word "table" specifies that the considered object is a piece of furniture with a given set of defining features. On this basis, it has been argued that in the process of word retrieval, common names benefit from the total activation coming from a number of related semantic representations, whereas proper names receive their activation only from the unique representation of the corresponding subject. ${ }^{1}$ From a neuropsychological point of view, this situation makes the link between an item in the semantic store and its proper name in the output lexicon especially fragile and liable to access problems.

$$
\begin{array}{r}
\text { L COHEN } \\
\text { F BOLGERT } \\
\text { S TIMSIT } \\
\text { J F CHERMANN } \\
\text { Service de Neurologie, } \\
\text { Hoppital de la Salpêtrière, Paris }
\end{array}
$$

Correspondence to: Dr Laurent Cohen, Service de Neurologie, Clinique Paul Castaigne, Hôpital de la Salpêtrière, $47 \mathrm{Bd}$ de l'Hôpital, 75651 Pari Cedex 13, France.

Burke DM, MacKay DG, Worthley JS, Wade E. On the tip of the tongue: what causes word finding failures in young and older adults? Fournal of Memory and Language 1991;30:542-79.

2 Cohen G, Faulkner D. Memory for proper names: Age differences in retrieval. British fournal of Developmental Psychology 1986;4:187-97.

3 Hodges JR, Salmon DP, Butters N. Recognition and naming of famous faces in Alzheimer's disease: A cognitive analysis. Neuropsychologia 1993;31:775-88.

4 McKenna P, Warrington EK. Testing for nominal dysphasia. I Neurol Neurosurg Psychiatry 1980;43:781-8.

5 Semenza C, Zettin M. Generating proper names: A case of selective inability. Cognitive Neuropsychology 1988;5:711-21.

6 Semenza C, Zettin M. Evidence from aphasia for the role of proper names as pure referring expressions. Nature 1989;342:678-9.

7 Lucchelli F, De Renzi E. Proper name anomia. Cortex 1992;28:221-30.

8 Carney R, Temple CM. Prosopanomia? A possible category-specific anomia for faces.
Cognitive Neuropsychology 1993;10:185-95.

9 Shallice T, Kartsounis LD. Selective impairment of retrieving people's names: A categoment of retrieving people's names: A catego-
ry specific disorder? Cortex 1993;29:281-91.

10 Damasio AR, Damasio H, Tranel D, Brandt JP. Neural regionalization of knowledge access: Preliminary evidence. Cold Spring Harb Symp Quant Biol 1991;55:1039-47.

11 Cambier J. Les aphasies sous-corticales. In Eustache F, Lechevalier B, eds. Langage et aphasie. Bruxelles: De Boeck Université, 1993:69-82.

12 McCarthy RA, Warrington EK. Cognitive neuropsychology. San Diego: Academic Press,
1990 . 